\title{
Leitfaden der Diätetik für Ärzte und Diätassistentinnen
}

Von

\section{Dr. Rudolf Wenger}

Assistent der I. Medizinischen Universitätsklinik Wien

Mit einem Geleitwort von

Professor Dr. Ernst Lauda

Vorstand der I.Medizinischen Universitätkklinik Wien

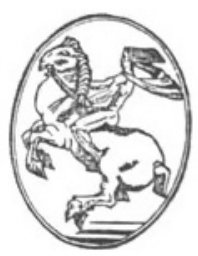

Springer-Verlag Wien GmbH 
ISBN 978-3-7091-3658-4 ISBN 978-3-7091-3657-7 (eBook)

DOI 10.1007/978-3-7091-3657-7

Alle Rechte,

insbesondere das der Übersetzung in fremde Sprachen, vorbehalten

Ohne ausdrückliche Genehmigung des Verlages

ist es auch nicht gestattet, dieses Buch oder Teile daraus

auf photomechanischem Wege (Photokopie, Mikrokopie)

$\mathrm{zu}$ vervielfältigen 


\section{Geleitwort}

Gab es noch vor etwa 30 Jahren nur eine Diätetik mit der Aufgabe, den Kranken trotz gegebener Schäden, die eine normale Ernährung unmöglich machten, doch noch optimal zu ernähren, wobei vor allem Magendarmkrankheiten, Durchfallkrankheiten, Darmdyspepsien oder Zustände mit schlechter Nahrungsausnutzung die wichtigste Domäne waren, oder eine Diätetik mit dem Ziel, eine gegebene Stoffwechselstörung, wie Diabetes, Gicht oder Fettsucht, diätetisch soweit möglich auszugleichen, Diätindikationen, die naturgemäß auch heute noch größte Bedeutung haben, so brachten die neuen Erkenntnisse auf dem Gebiete der Hypo- und Avitaminosen einerseits und die neuen Anschauungen über die Bedeutung der Diät bei Herz-, Leberparenchymerkrankungen, bei Wassersucht aller Art, bei Nierenerkrankungen anderseits, um nur die wichtigsten zu nennen, und schließlich auch über die mögliche Heilkraft einer Diät, etwa bei Tuberkulose wie die nach Gerson, neue Forderungen im Sinne einer verschiedenartigen Heilkost. Diese Forschungsrichtung führte zu früher fast nicht für möglich gehaltenen Erfolgen, wie etwa die der Leberdiät beim Morbus Biermer, allerdings aber auch zu Auswüchsen, die die Diätetik zum Teil sogar diskreditierten, wobei wir insbesondere die Heilkost beim Karzinom im Auge haben, wenigstens solange die sogenannte „krebsfeindliche Diät““ nicht eingehender wissenschaftlich studiert ist und beweisende Argumente für ihre Berechtigung beigebracht sind. Es ist hier nicht der Ort, dieses Problem näher auszuführen, es sollte auf diese Verhältnisse unter Hinweis auf einige neue Richtungen der Diätetik nur aufmerksam gemacht werden, um zu zeigen, daß die moderne Medizin der Diätetik immer größere Bedeutung beigelegt und den Indikationsbereich für eine Diätetik immer weiter gesteckt hat. Auch der Praktiker begegnet dem Problem der Diätetik immer häufiger. 
Aus diesen Ausführungen ist zu ersehen, daß im Rahmen der verschiedenartigen diätetischen Bestrebungen nur eine kritische Einstellung zu Diätfragen erfolgversprechend sein kann. Um richtige Diätetik zu betreiben, genügt es nicht, verschiedene Speisezettel für verschiedene Krankheiten zu kennen, Voraussetzung sind immer auch die Kenntnisse der Grundtatsachen der Verdauungsphysiologie und auch ein spezielles Wissen über die Möglichkeit der diätetischen Beeinflußbarkeit verschiedener Krankheiten.

Vom Praktiker wie von der Diätassistentin als Beraterin einer Diätküche werden aber nicht nur die Kenntnis einer bestimmten Kostform, von Speisezusammenstellungen und von Kochrezepten, sondern auch fundierte Kenntnisse aus Verdauungs- und Ernährungsphysiologie und -pathologie vorausgesetzt werden müssen:

Die Diätetik liegt hierzulande und in Europa überhaupt noch vielfach im argen. Daß Frischoperierte, wie man nicht selten erlebt, am Tag nach der Operation eine Normalkost erhalten oder daß sie fasten und dursten müssen, sind keine medizinisch richtigen Lösungen.

Dem Leiter der Diätassistentinnenschule meiner Klinik, Assistent Dr. R. Wenger, war ein diätetischer Leitfaden, welcher die angedeuteten Kenntnisse vermittelt, beim Unterricht seit langem ein Bedürfnis. Wenn er bei der gebotenen Kürze dieses Leitfadens auch auf alle Details einer Diätetik nicht eingehen konnte, so gibt der Leitfaden aber doch nicht nur die Diätetik im engeren Sinne, sondern er vermittelt für eine richtige Diätetik auch die notwendigen Kenntnisse. Ich bin überzeugt, daß Wengers Leitfaden seinen Weg machen wird und daß er Ärzten, Studenten, Diätassistentinnen und allen, die sich ernsthaft mit praktischer Diätetik beschäftigen, eine wertvolle Hilfe sein wird.

Wien, im Juni 1955

Ernst Lauda 


\section{Vorwort}

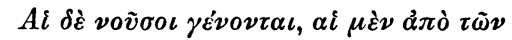

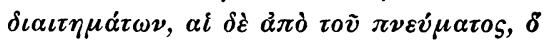
$\varepsilon \sigma \alpha \gamma \delta \dot{\alpha} \varepsilon v o \iota \zeta \tilde{\omega} \mu \varepsilon v$.

Hippokrates

Das vorliegende Buch soll sowohl dem Arzt bzw. dem Medizinstudenten als auch der Diätassistentin als knapper Leitfadèn für diätetische Behandlungsmethoden dienen. Es wurde deshalb in allen Fällen versucht, die Zweckmäßigkeit der empfohlenen diätetischen Maßnahmen auf dem Boden der Physiologie und Pathologie darzustellen.

Um den Umfang des Buches beschränkt halten zu können, wurden bei den einzelnen Erkrankungen meistens nur allgemeine Richtlinien gegeben. Von genauen quantitativen Angaben wurde in den meisten Fällen abgesehen.

Die Rücksichtnahme auf den Umfang der Arbeit brachte es auch mit sich, daß manches nur in schematischer Form dargestellt werden konnte. Arzt und Diätassistentin sollen im einzelnen Falle die aufgezeigten Grundregeln in individualisierender Art anwenden. Wenn es möglich ist, soll der Patient individuell beraten werden. Genaue, dem Einzelfall angepaßte Verordnungen sind beim Patienten mit Recht mehr beliebt als Merkblätter, die man ihm in die Hand drückt. Bei den Besprechungen der einzelnen Erkrankungen wurde auch stets auf die Wichtigkeit eines entsprechenden Kostaufbaues im Verlaufe der Erkrankung hingewiesen. Hier konnten allerdings wiederum meistens nur allgemeine Angaben gemacht werden, so daß es eine wichtige Aufgabe des Arztes bleibt, im Einzelfall die Geschwindigkeit des Kostaufbaues - je nach dem Fortschreiten der klinischen Besserung - zu bestimmen.

Es ist wohl selbstverständlich, daß bei der Auswahl der bei den einzelnen Erkrankungen empfohlenen Speisen der Einfluß 
der Wiener Küche unverkennbar ist; es ist unser besonderes Anliegen, die Methoden dieser Küche dem Wohl des Diätpatienten noch weiter dienstbar zu machen. Darüber hinaus werden jedoch auch Küchenmethoden und Nahrungsmittel, mit denen der Leser meist nur in anderen Ländern Europas oder in tubersee in Berührung kommt, besprochen. Dasselbe gilt für einzelne Erkrankungen. Derartige schlaglichtartige Hinweise schienen oft notwendig, um eine Frage in allen ihren Zusammenhängen besprechen zu können. Außerdem soll dadurch die Beratung ausländischer Patienten, die in unseren Kliniken, Sanatorien, bzw. in der Sprechstunde Hilfe suchen, unterstützt werden. Schließlich mag es in manchen Fällen dem Arzt oder der Diätassistentin, die in fremde Länder gehen, die Umstellung auf die dortigen Verhältnisse erleichtern.

Ein Gedanke zieht sich wie ein roter Faden durch das vorliegende Buch: Die Nahrung stellt für den Patienten, vor allem für den an einer lange dauernden Erkrankung leidenden, oft eine der wenigen oder sogar die einzige Freude dar, die ihm der trostlos erscheinende Alltag zu bieten hat. Es bedarf der verständnisvollen Zusammenarbeit zwischen Arzt, Diätassistentin und Angehörigen, bzw. dem Küchenpersonal und den Schwestern. Nicht nur bei der Auswahl der Speisen soll, soweit es möglich ist, auf die Wünsche des Patienten Rücksicht genommen werden, auch beim Anrichten der Mahlzeiten soll auf ein geschmackvolles Äußeres großer Wert gelegt werden.

Der Kochkunst fallen im Rahmen der Diätetik besondere Aufgaben zu. Oft müssen wir versuchen, den Patienten trotz schlechtem oder völlig darniederliegenden Appetit zu einer ausreichenden Nahrungsaufnahme zu bewegen. Wohlschmeckende und appetitlich angerichtete Speisen sind dazu die wichtigste Voraussetzung. Oft macht es auch die mit Rücksicht auf die Erkrankung beschränkte Auswahl der zur Verfügung stehenden Nahrungsmittel oder Kochmethoden sehr schwierig, geschmackvolle Speisenzusammenstellungen zu bereiten. Es ist jedoch häufig verblüffend, was eine gute und einfallsreiche Köchin auch noch im Rahmen einer sehr weitgehend beschränkten Diät herzustellen vermag.

In manchen Kapiteln der Diätetik scheint eine „Umwertung der Werte" vor sich zu gehen, so z. B. bei der diätetischen Be- 
handlung der Lebererkrankungen, der Gicht oder der Nierensteinerkrankungen. Dort, wo die neueren Ansichten noch nicht genügend gefestigt erschienen, wurden sie zwar erwähnt, es wurde jedoch sonst noch ein eher konservativer Standpunkt eingenommen. Wenn die Besprechung der Diätetik einzelner, selten vorkommender Erkrankungen einen unverhältnismäßig großen Raum einzunehmen scheint, ist dies darauf zurückzuführen, daß gerade derartige Diäten häufig verhältnismäßig kompliziert sind, bzw. daß gerade deren Darstellung höherer didaktischer Wert zukommt.

Meinem verehrten Lehrer, Herrn Prof. Dr. Ernst Lauda, möchte ich für seine wertvolle Beratung und Unterstützung bei der Herausgabe dieses Buches besonders danken. Dem SpringerVerlag und seinem Inhaber, Herrn Otto Lange, der die Herausgabe des Buches ermöglichte, möchte ich für die exakte Ausführung aufrichtigen Dank sagen.

Wien, im Juni 1955 


\section{Inhaltsverzeichnis}

I. Physiologische Vorbemerkungen . . . . . . . . . . . 1

1. Ernährungsphysiologie . . . . . . . . . . . . . . 1

2. Physiologie der Nahrungsaufnahme . . . . . . . . . . . 6

3. Mineralstoffe . . . . . . . . . . . . . . . . . . 8

4. Vitamine . . . . . . . . . . . . . . . . . . . 11

a) Allgemeines . . . . . . . . . . . . . . . . . . . . . . 11

b) Wasserlösliche Vitamine . . . . . . . . . . . 14

c) Fettlösliche Vitamine . . . . . . . . . . . . . 19

II. Erkrankungen des Magendarmtraktes . . . . . . . . . 22

1. Erkrankungen der Mund- und Rachenhöhle sowie der Speiseröhre . . . . . . . . . . . . . . . . . . 22

2. Erkrankungen des Magens, Magenschonkost . . . . . 22

3. Ulkuskrankheit . . . . . . . . . . . . . . . . 27

4. Ulkusblutung . . . . . . . . . . . . . . . . . 31

5. Pylorusstenose . . . . . . . . . . . . . . . . . . . . . 32

6. Zustand nach Magenresektion, Stumpfgastritis . . . . 32

7. Magenkarzinom . . . . . . . . . . . . . . . . 33

8. Allgemeine Gesichtspunkte bei Dünndarmerkrankungen. Die „aufgeschlossene“ Kost . . . . . . . . . . . 34

9. Akute Enteritis . . . . . . . . . . . . . . . . 38

10. Chronische Enteritis . . . . . . . . . . . . . 39

11. Sprue . . . . . . . . . . . . . . . . . . 40

12. Cöliakie . . . . . . . . . . . . . . . . . . . 41

13. Typhus . . . . . . . . . . . . . . . . . . . 41

14. Dysenterie . . . . . . . . . . . . . . . . . . 43

15. Colitis, Dickdarmschonkost . . . . . . . . . . . . 43

16. Gärungswidrige Kost . . . . . . . . . . . . . . 46

17. Fäulniswidrige Kost . . . . . . . . . . . . . . 47

18. Meteorismus, Flatulenz . . . . . . . . . . . . . 48

19. Chronische Obstipation . . . . . . . . . . . . . 48

a) Atonische Form . . . . . . . . . . . . . . . . . 49

b) Spastische Form . . . . . . . . . . . . . . 50

20. Gastroptose, Enteroptose . . . . . . . . . . . . . 51

III. Künstliche Ernährung . . . . . . . . . . . . . . 51

1. Sondenernährung . . . . . . . . . . . . . . . . 51

2. Ernährung durch Magen- oder Darmfisteln . . . . . . 53

3. Rektale Ernährung . . . . . . . . . . . . . . . 53

4. Parenterale Ernährung . . . . . . . . . . . . . 54 
IV. Lebererkrankungen . . . . . . . . . . . . . . . . 55

1. Allgemeines . . . . . . . . . . . . . . . . . . 55

2. Akute Hepatitis . . . . . . . . . . . . . . . . 57

3. Leberzirrhose . . . . . . . . . . . . . . . . . 57

4. Leberschonkost . . . . . . . . . . . . . . . . . 58

5. „Posthepatitisches Syndrom“ . . . . . . . . . . . 60

V. Gallenblasenerkrankungen . . . . . . . . . . . . . . 60

VI. Störungen der äußeren Sekretion des Pankreas . . . . . 66

VII. Diabetes mellitus . . . . . . . . . . . . . . . . 67

VIII. Fettleibigkeit und Fettsucht . . . . . . . . . . . 80

IX. Unterernährung und Magersucht . . . . . . . . . . 91

X. Herz- und Gefäßkrankheiten . . . . . . . . . . . . . 94

1. Allgemeines . . . . . . . . . . . . . . . . . . 94

2. Kochsalz-(natrium-) arme Kost . . . . . . . . . . . . 95

3. Andere diätetische Maßnahmen bei Herzinsuffizienz und bestimmten kardialen Erkrankungen . . . . . . . . 99

4. Essentielle Hypertonie . . . . . . . . . . . . . . 101

5. Arteriosklerose . . . . . . . . . . . . . . . . . 102

Xl. Nierenerkrankungen . . . . . . . . . . . . . . . 105

1. Allgemeines . . . . . . . . . . . . . . . . . . 105

2. Akute Nephritis (Strenge Nierenschonkost) . . . . . . 107

3. Chronische Nephritis . . . . . . . . . . . . . 108

a) Milde Nierenschonkost . . . . . . . . . . . . 108

b) Chronische Nephritis mit Odemen . . . . . . . . 109

c) Chronische Nephritis mit starker Blutdruckerhöhung . 109

d) Chronische Nephritis mit stärkerer Reststickstoffsteigerung. Urämie . . . . . . . . . . . . . . . 109

4. Nephrosklerose mit Niereninsuffizienz . . . . . . . . 111

5. Nephrose . . . . . . . . . . . . . . . . . . . 112

6. Nierentuberkulose . . . . . . . . . . . . . . . . 112

XII. Erkrankungen des Nierenbeckens und der ableitenden Harnwege . . . . . . . . . . . . . . . . . . . 113

1. Pyelitis . . . . . . . . . . . . . . . . . . . . 113

2. Nierensteinerkrankungen . . . . . . . . . . . . . 114

a) Allgemeines . . . . . . . . . . . . . . . . . 114

b) Oxalatsteine . . . . . . . . . . . . . . . 115

c) Phosphatsteine . . . . . . . . . . . . . . . . 117

d) Harnsäuresteine . . . . . . . . . . . . . . 118

e) Cystinurie . . . . . . . . . . . . . . . . . . . 118

f) Alkaptonurie . . . . . . . . . . . . . . . . 119

XIII. Gicht. Purinarme Kost . . . . . . . . . . . . . . 119

XIV. Endokrine Erkrankungen, Hormontherapie . . . . . . 122

1. Addisonsche Krankheit. Kaliumarme Kost . . . . . . 122

2. Hyperthyreose . . . . . . . . . . . . . . . . 123

3. Funktionelle Hypoglykämie („Hyperinsulinismus“) . . . 124 
4. Diätetische Maßnahmen während der Durchführung einer ACTH- oder Cortison-Therapie . . . . . . . . . . . 124

5. Kalziumreiche (Rekalzifikations-) Diät . . . . . . . 125 $X V$. Anämien . . . . . . . . . . . . . . . . . . . 125 $X V I$. Infektionskrankheiten . . . . . . . . . . . . . . 128 $X V I I$. Chronische Erkrankungen und Neoplasmen . . . . . . 129 XVIII. Prä- und postoperative Diäten. Diät bei chirurgischen Erkrankungen . . . . . . . . . . . . . . . . 130

1. Allgemeines . . . . . . . . . . . . . . . . . . 130

2. Diät nach Laparotomien . . . . . . . . . . . . 131

3. Diät bei Colostomie . . . . . . . . . . . . . . . 133

4. Diät bei Operationen am Mastdarm . . . . . . . . . 133

5. Diät bei chirurgischen Eingriffen und gleichzeitiger Leberschädigung . . . . . . . . . . . . . . . . . . 134

6. Flüssige, bzw. halbflüssige, schlackenarme Kost . . . . 134

7. Feste, schlackenarme Kost . . . . . . . . . . . . 134

8. Erweiterte schlackenarme Kost . . . . . . . . . . . 135

XIX. Nahrungsmittelallergien . . . . . . . . . . . . . . 135

1. Allgemeines . . . . . . . . . . . . . . . . . . 135

2. Methoden der Erkennung von Nahrungsmittelallergenen . 137

a) Testmahlzeit ... . . . . . . . . . . . . . . 137

b) Eliminationsdiät . . . . . . . . . . . . . . . 138

3. Diätetische Therapie der Nahrungsmittelallergien . . . 140

$X X$. EiweiBreiche Kost . . . . . . . . . . . . . . . . 142

XXI. Die Rohkost und ihr nahestehende Diätformen . . . . 143

1. Rohkost . . . . . . . . . . . . . . . . . . . 143

2. Saftkost . . . . . . . . . . . . . . . . . . . 145

3. Erweiterte Rohkost . . . . . . . . . . . . . . . 145

4. Obstdiät . . . . . . . . . . . . . . . . . . . 146

5. Sauermilch-Obstdiät . . . . . . . . . . . . . . . 146

6. Gerson-Diät . . . . . . . . . . . . . . . . . . 146

7. Die Sauerbruch-Hermannsdörfersche Kost . . . . . . 147

XXII. Säuernde und alkalisierende Kost . . . . . . . . . 147

1. Allgemeines . . . . . . . . . . . . . . . . . . 147

2. Säuernde Kost . . . . . . . . . . . . . . . . . 148

3. Alkalisierende Kost . . . . . . . . . . . . . . . 150

XXIII. Probekostformen . . . . . . . . . . . . . . . 151

1. Schmidt-Straßburgersche Probekost . . . . . . . 151

2. Kalziumarme Kost . . . . . . . . . . . . . . . 151

3. Kaliumreiche, kochsalzarme Probekost nach Cutler, Power und Wilder . . . . . . . . . . . . . . . . . . 151

Kalorien- und Nährstofftabelle . . . . . . . . . . . 153

Literaturverzeichnis . . . . . . . . . . . . . . . 157

Sachverzeichnis . . . . . . . . . . . . . . . 161 